Nota Científica

(Short Communication)

\title{
REPRODUCCIÓN DEL FALSO VAMPIRO LANUDO CHROTOPTERUS AURITUS (CHIROPTERA: PHYLLOSTOMIDAE) EN UN BOSQUE TROPICAL HÚMEDO DE LA COSTA PACÍFICA DE GUATEMALA
}

\author{
REPRODUCTION OF THE WOOLLY FALSE VAMPIRE BAT CHROTOPTERUS \\ AURITUS (CHIROPTERA: PHYLLOSTOMIDAE) IN A HUMID TROPICAL FOREST \\ OF THE PACIFIC COAST OF GUATEMALA
}

\author{
Rocío A. ÁlVAREZ-YAX, ${ }^{1}$ ANa H. GOMEZ- LEMUS, ${ }^{1}$ JULIA S. HERNÁNDEZ-FUENTES, ${ }^{1}$ \\ Andrea P. JUAREZ-BOLAÑOS, ${ }^{1}$ KATTY J. PEREZ-QUAN, ${ }^{1}$ DONALd G. TIJERINO-ESCOBAR, ${ }^{1}$ \\ MElissa VILLATORO-CASTAÑEDA ${ }^{1}$ Y DANIEL ARIANO-SÁNCHEZ ${ }^{2, *}$ \\ ${ }^{1}$ Departamento de Biología, Universidad del Valle de Guatemala, 11 Calle 15-79 zona 15 V.H. III, Ciudad de \\ Guatemala, Guatemala. \\ ${ }^{2}$ Centro de Estudios Ambientales y Biodiversidad, Instituto de Investigaciones, Universidad del Valle de \\ Guatemala, 18 avenida 11-95 zona 15 V.H. III, Ciudad de Guatemala, Guatemala \\ *Autor para correspondencia:<dariano@uvg.edu.gt>
}

Recibido: 10/07/2017; aceptado: 30/10/2017; publicado en línea: 16/03/2018

Editor responsable: Vinicio Sosa

Álvarez-Yax, R. A., Gómez-Lemus, A. H., Hernández-Fuentes, J. S., Juárez-Bolaños, A. P., Pérez-Quan, K. J., Tijerino-Escobar, D. G., Villatoro-Castañeda, M. y Ariano-Sánchez, D. (2018) Reproducción del falso vampiro lanudo Chrotopterus auritus (Chiroptera: Phyllostomidae) en un bosque tropical húmedo de la costa pacífica de Guatemala. Acta Zoológica Mexicana (n.s), 34(1), 1-3.

RESUMEN. Documentamos una hembra de Chrotopterus auritus en estado de lactancia con su cría presente en la parte ventral de su cuerpo. El espécimen fue capturado con una trampa de arpa en un bosque tropical húmedo de la costa del Pacífico de Guatemala, dentro de la reserva natural privada Finca Patrocinio, Quetzaltenango, Guatemala el 29 de mayo de 2017. Hay poca información sobre la biología de esta especie, especialmente en Guatemala, lo que refleja la importancia de nuevos estudios sobre el comportamiento silvestre de esta especie.

El falso vampiro lanudo Chrotopterus auritus, es uno de los murciélagos más grandes del neotrópico. Mide aproximadamente $78-83 \mathrm{~cm}$ y pesa $75-96 \mathrm{~g}$ y se alimenta principalmente de artrópodos grandes, aves pequeñas y pequeños mamíferos como ratones (Witt, 2010). Se encuentra desde Argentina hasta el sur de México. Habita en bosques lluviosos tropicales, deciduos y nubosos. Se han
Álvarez-Yax, R. A., Gómez-Lemus, A. H., Hernández-Fuentes, J. S., Juárez-Bolaños, A. P., Pérez-Quan, K. J., Tijerino-Escobar, D. G., Villatoro-Castañeda, M., \& Ariano-Sánchez, D. (2018) Reproduction of the woolly false vampire bat Chrotopterus auritus (Chiroptera: Phyllostomidae) in a humid tropical forest of the pacific coast of Guatemala. Acta Zoológica Mexicana (n.s), 34(1), 1-3.

ABSTRACT. We documented one female Chrotopterus auritus in a lactation state with her calf present in the ventral part of her body. The specimen was captured with a harp trap in a humid tropical forest of the Pacific coast of Guatemala, within private natural reserve Finca Patrocinio, Quetzaltenango, Guatemala on May 29th, 2017. There is little information about this species' biology, especially within Guatemala, which reflects the importance of further studies on the wild behavior of this species.

registrado ejemplares en México, Perú, Bolivia, Brasil, Argentina, Venezuela y Paraguay, pero existen muy pocos reportes detallados para Guatemala (Medellín, 1989). La información de la biología reproductiva de $C$. auritus es escasa, proviniendo principalmente de la observación de ejemplares en cautiverio (Esbérard, et al., 2006).

Las escasas observaciones disponibles sobre la repro- 
ducción de esta especie en la naturaleza, reportan un patrón reproductivo monoestral con un período de gestación cercano a los 7 meses, que varía de acuerdo a la localidad geográfica (Taddei, 1976). Las colonias en cautiverio, bajo fotoperiodos naturales y a la misma latitud de ocurrencia, muestran una periodicidad similar (Esbérard, et al., 2006).

Entre los meses de febrero a junio de 2017 se realizó una Evaluación Ecológica Rápida (EER) en la Reserva Natural Privada Finca Patrocinio (RNP-FP), ubicada en el departamento de Quetzaltenango, Guatemala. La RNPFP es una finca privada de 140 hectáreas de las cuales el $65 \%$ está dedicado al policultivo agrícola, con plantaciones de macadamia, rambután, café, cacao orgánico y aguacate. E1 35\% restante es destinado a la conservación de bosque tropical húmedo. La RNP-FP está ubicada entre los 800-950 metros sobre el nivel del mar (msnm). Como parte de esta EER se colocó una trampa arpa (G7 harp trap, Bat Conservation and Management, Inc.) a medio metro de distancia de la cavidad de un árbol de chilamate (Ficus insípida de $160 \mathrm{~cm}$ de diámetro a la altura de pecho), dentro de un parche de bosque tropical húmedo de aproximadamente 20 hectáreas. Al examinar la cavidad interna del tronco de este árbol se observó una colonia de cinco individuos de $C$. auritus dentro de la misma, estaban a aproximadamente 6 metros de altura colgando de raíces de plantas trepadoras que penetraban en la cavidad del tronco. La trampa arpa se dejó puesta dos días en un horario de 0000 a $0500 \mathrm{hrs}$. Esta colonia de C. auritus había sido documentada en el 2014 en una EER previa en la RNP-FP (Alarcón et al., 2014) y estaba conformada por cuatro ejemplares, un macho y tres hembras (Fig. 1) que habitaban la misma cavidad del árbol de F. insipida (D. Ariano-Sánchez, obs. pers.).

Mediante la trampa arpa capturamos a una hembra lactante de C. auritus con una cría presente en la parte ventral de su cuerpo a las $0300 \mathrm{hrs}$ del 28 de mayo de 2017 (Fig. 2), lo que nos permitió establecer que dicha colonia estaba formada en el 2017 por seis ejemplares en total, incluyendo a esta hembra lactante con su cría. El ejemplar fue capturado a inicios de la época lluviosa. Tomando en cuenta que la cría tenía casi el mismo tamaño que la hembra, calculamos que la misma tendría un mes de nacida, de acuerdo a las estimaciones de proporción de tamaño entre cría y madre realizadas en cautiverio por Esbérard et al. (2006). Estos mismos autores reportan un período de gestación de aproximadamente siete meses, por lo que estimamos que el ciclo reproductivo de $C$. auritus en la costa pacífica de Guatemala constaría de apareamientos

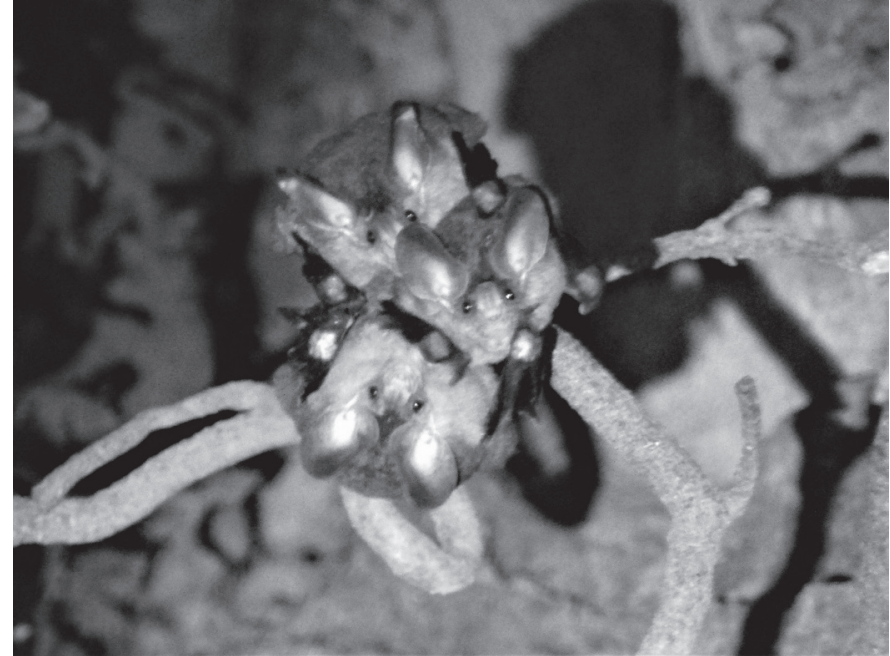

Figura 1. Colonia de falso vampiro lanudo Chrotopterus auritus (Chiroptera: Phyllostomidae) observada en 2014 dentro de cavidad de árbol en un bosque húmedo del pacífico de Guatemala (Foto D. Ariano-Sánchez).

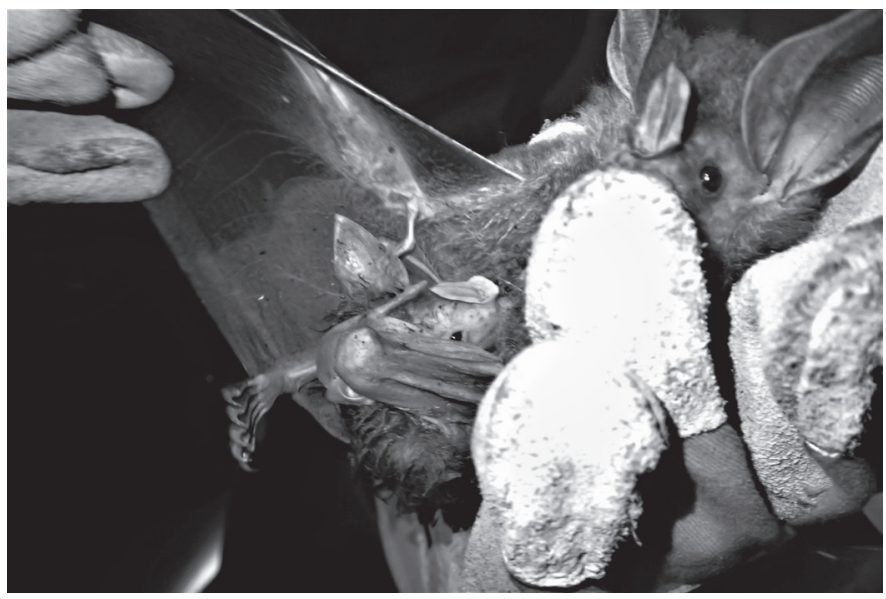

Figura 2. Hembra lactante de falso vampiro lanudo Chrotopterus auritus (Chiroptera: Phyllostomidae) capturada con cría en brazos en 2017 al salir de cavidad de árbol en un bosque húmedo del pacífico de Guatemala (Foto M. Villatoro-Castañeda).

entre los meses de agosto y septiembre, con nacimientos de las crías entre los meses de abril y mayo.

AGRADECIMIENTOS. Al Departamento de Biología de la Universidad del Valle de Guatemala, por brindarnos el equipo de investigación, a la RNP Finca Patrocinio y a nuestro equipo de trabajo por el apoyo brindado durante nuestras giras de campo. Agradecemos también los comentarios realizados por dos árbitros anónimos que revisaron la nota y mejoraron la redacción y estilo de la misma. La investigación se realizó de acuerdo a la Licencia de Investigación 00554 de CONAP. 


\section{LITERATURA CITADA}

Alarcón, M., Arrivillaga, C., García, A., Gelera, R., Gonzalez, S., Mármol, A., Martínez, A., Moller, D., Moller, G., Sactic, M., Sagastume, V., Viteri, M., Braeuner, M, \& Ariano-Sánchez, D. (2014) Evaluación ecológica rápida de la Reserva Natural Privada Finca Patrocinio, El Palmar, Quetzaltenango. Universidad del Valle de Guatemala, Guatemala. 102 pp.

Esbérard, C. E., Motta, A.G., Almeida, J.C., Ferreira, L.C. \& Costa, L. M. (2006) Reproduction of Chrotopterus auritus (Peters) in captivity (Chiroptera, Phyllostomidae). Brazilian Journal of Biology, 66 (3), 995-956.

Medellín, R. (1989) Chrotopterus auritus. Mammalian Species, 343, 1-5.

Taddei, A. (1976) The reproduction of some Phyllostomidae (Chiroptera) from the northeastern region of the state of Sao Paulo. Boletin de Zoología de la Universidad de Sao Paulo, 1, 313-330.

Witt, A. (2010) Hábitos alimenticios de Chrotopterus auritus (Chiroptera, Phyllostomidae). Mastozoología Neotropical, 17(2), 353360. 